\title{
Volume EM of Pathological Deposits - Array Tomography and FIBSEM Nanotomography on Weakly Metalized Routine Samples
}

\author{
Irene Wacker ${ }^{1}$, Carsten Dittmayer ${ }^{2}$, Joseph A. Schroeder ${ }^{3}$ and Rasmus R. Schröder ${ }^{1,4}$ \\ ${ }^{1}$ Cryo EM, Centre for Advanced Materials, Universität Heidelberg, Germany \\ ${ }^{2}$ Department of Neuropathology, Charité-Universitätsmedizin, Berlin, Germany \\ ${ }^{3}$ Central EM-Lab, Pathology Department, Uniklinikum Regensburg, Germany \\ ${ }^{4}$ Cryo EM, BioQuant, Universitätsklinikum Heidelberg, Germany
}

Advanced SEM imaging techniques, such as array tomography (AT) for serial sections and focused ion beam (FIB)-based nanotomography working directly on sample blocks, made imaging of large volumes at ultrastructural resolution (volume EM) feasible [1]. For high image contrast and good conductivity a number of block-staining methods have been developed, impregnating the sample with large amounts of different heavy metals [2]. Contrary to that, routine samples from the pathology laboratory are usually prepared according to standardized protocols relying mostly on moderate concentrations of $\mathrm{OsO}_{4}$ as a contrasting agent. Here we show how volume EM may be applied to such weakly stained pathology samples, using as examples intracellular inclusions (nemaline rods) in nemaline-related myopathy [3] and pathognomic extracellular deposits (granular osmiophilic material, GOM) in CADASIL disease [4]. For AT, serial sections of a muscle biopsy were placed on silicon (Fig. 1 A) and post-stained with uranyl acetate and lead citrate. Cells with nemaline rods were identified with a light microscope (LM, Fig. 1 B), and imaged in the SEM using back-scattered electrons (Fig. $1 \mathrm{C}$ ) at a resolution that allows visualization of the disarrangement of the sarcomeric structure in the region of the rods (Fig. 1 D). Segmentation of rods in 3D reconstructions of image stacks (Fig. 1 E) revealed features such as branched rods (Fig. 1 F). Since the anisotropic Z-resolution obtained by AT is not suited for the small CADASIL GOMs we applied FIBSEM nanotomography. Perivascular smooth muscle cells carrying deposits were targeted in two steps: A small arteriole in a skin biopsy (Fig. 2 A) was mapped by intermediate resolution AT to identify GOM-positive cells. This map was overlaid on the block face of the Pt-sputtered block (Fig. 2 B). The resulting image stacks showed less contrast due to the low metal content (Fig. 2 C), but after simple image processing routines contrast was sufficient to allow segmentation of the GOMs (Fig. 2 D). Comparing AT with FIBSEM nanotomography, the latter has the advantage of a better resolution in Z, isotropic voxels of $5 \times 5 \times 5 \mathrm{~nm}$ are feasible for small volumes such as parts of cells. With suitable imaging regimes even blocks with relatively low metal content (e.g. $1 \% \mathrm{OsO}_{4}$ ) produce data adequate for segmentation. The possibility to post-stain the section arrays is a decided plus for AT. In addition, here the material is not destroyed by the imaging process (as is the case for FIB milling) and is available for further imaging rounds. Currently we are exploring a variety of alternative post stains such as lanthanide salts for their potential to confer contrast and conductivity to even less metallized samples.

\section{References:}

[1] CJ Peddie, LM Collinson, Micron 61 (2014), p. 9

[2] JC Tapia, et al., Nat Protoc 7 (2012), p. 193

[3] E Malfatti et al., Acta Neuropathol Commun 2 (2014), p. 44

[4] Morroni et al., PLoS One 8 (2013), e65482

[5] We thank L. Veith and M. van Ark for technical support and the Federal Ministry of Education and Research for MorphiQuant-3D grant FKZ 13GW0044. 

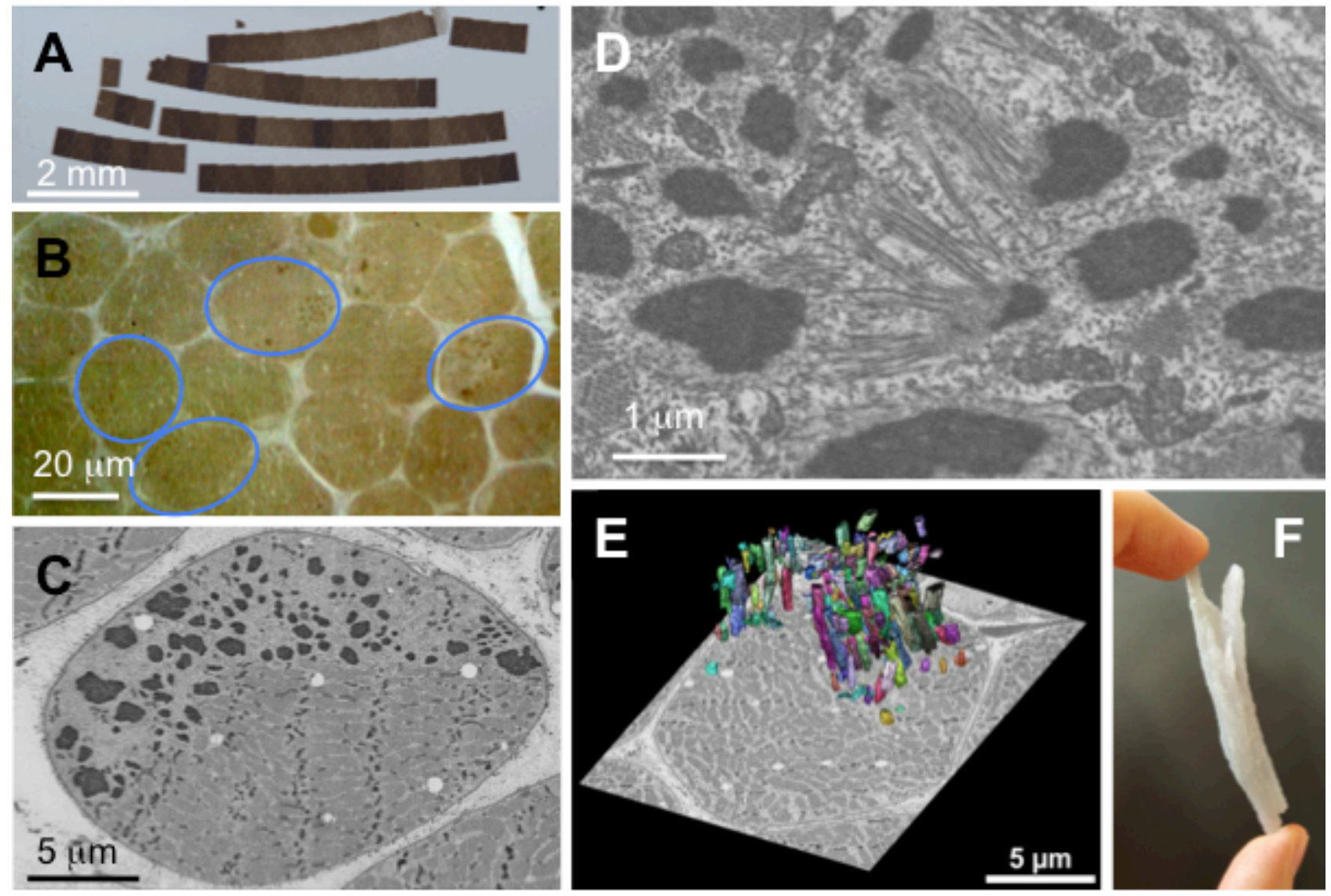

Figure 1. Nemaline myopathy: Array of serial sections on silicon wafer (A), selection of muscle cells containing nemaline rods (blue circles) using LM (B), overview of one muscle cell recorded with $5 \mathrm{~nm}$ image pixels in the SEM (C), electronic zooming to detail (D), segmentation of nemaline rods (E), 3D print of one $\operatorname{rod}(\mathrm{F})$.
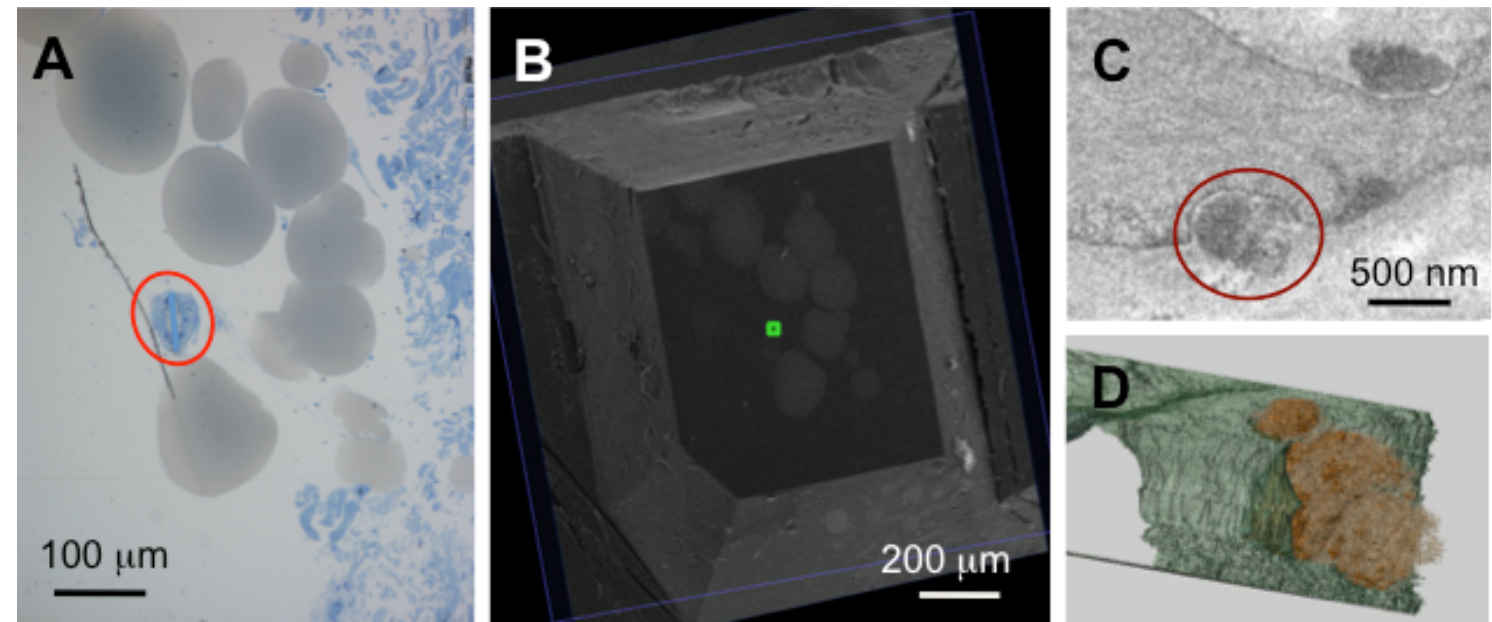

Figure 2. CADASIL: LM of skin biopsy, arteriole (red circle) surrounded by fat cells (A). Pt-coated sample block in FIBSEM, green square marks position of arteriole (B). Raw data - one slice from nanotomography run $(\mathrm{C})$, circle indicates GOM shown in 3D with plasma membrane segmented in green, GOM in brown (D). 